\title{
ATRIBUTOS QUÍMICOS E MICROBIANOS DO SOLO DE ÁREAS EM PROCESSO DE DESERTIFICAÇÃO NO SEMIÁRIDO DE PERNAMBUCO ${ }^{(1)}$
}

\author{
Carolina Malala Martins ${ }^{(2)}$, Izabel Cristina de Luna Galindo(3), \\ Edivan Rodrigues de Souza ${ }^{(4)}$ \& Henrique Arruda Poroca ${ }^{(5)}$
}

\section{RESUMO}

Devido ao avanço das áreas em processo de degradação decorrente de causas naturais e, ou, influências antrópicas, faz-se necessária a utilização de ferramentas, preferencialmente de baixo custo, que indiquem os estádios dessa degradação, a fim de viabilizar um manejo adequado e a recuperação dessas áreas. Desse modo, o objetivo desta pesquisa foi analisar a variabilidade de atributos químicos e microbianos de solos, visando a utilizá-los como indicadores de processos de desertificação, em áreas sob níveis crescentes de degradação na região semiárida do Estado de Pernambuco. Foram amostrados três ambientes: conservado, moderadamente degradado e intensamente degradado, no município de FlorestaPE. Em cada ambiente foram escolhidas quatro áreas de amostragens e efetuadas sete coletas em cada um delas, totalizando 28 pontos amostrados por ambiente. Foram coletadas amostras de solo na profundidade de $0-10 \mathrm{~cm}$, nos períodos seco e chuvoso. Realizaram-se análises químicas (pH, bases trocáveis, acidez potencial, C orgânico do solo, estoque de $\mathrm{C}, \mathrm{P}$ disponível e capacidade de troca de cátions) $\mathrm{e}$ microbianas (C da biomassa microbiana, respiração basal, quocientes microbiano e metabólico) do solo, além da caracterização física (textura e densidade do solo). Os dados foram analisados adotando-se métodos de análises de componentes principais e teste t. Verificou-se o efeito da sazonalidade para os atributos do solo, sendo essa mudança mais perceptível no ambiente degradado. Com as análises de componentes principais, foi possível destacar alguns atributos mais sensíveis ao

\footnotetext{
(1) Parte da Dissertação de Mestrado do primeiro autor apresentada ao Programa de Pós-graduação em Ciência do Solo da Universidade Federal Rural de Pernambuco - UFRPE. Recebido para publicação em abril de 2009 e aprovado em outubro de 2010.

${ }^{(2)}$ Professora Assistente do Departamento de Ciências Ambientais e Tecnológicas, Universidade Federal Rural do Semiárido UFERSA. CEP 59625-900 Mossoró (RN). E-mail: carolmalala@ufersa.edu.br

${ }^{(3)}$ Professora Associada do Departamento de Agronomia, Universidade Federal Rural de Pernambuco - UFRPE. CEP 52171-900 Recife (PE). E-mail: iclgalindo@uol.com.br

(4) Professor Adjunto do Departamento de Agronomia, UFRPE. E-mail: edivan@depa.ufrpe.br

(5) Engenheiro-Agrônomo do Ministério do Desenvolvimento Agrário - MDA/AP. CEP 68908-610 Macapá (AP). E-mail: henrique.poroca@mda.gov.br
} 


\begin{abstract}
avanço da degradação, como o $\mathrm{C}$ da biomassa microbiana do solo, a acidez potencial e a saturação por bases, tanto no período seco como no chuvoso. Desse modo, esses atributos podem ser utilizados como indicadores do nível de degradação do solo.
\end{abstract}

Termos de indexação: degradação do solo, matéria orgânica, qualidade do solo.

\author{
SUMMARY: MICROBIOLOGICAL AND CHEMICAL SOIL PROPERTIES IN \\ DESERTING AREAS IN THE SEMIARID REGION OF \\ PERNAMBUCO STATE, BRAZIL
}

\begin{abstract}
To analyze the process of degradation caused by natural or anthropogenic influences tools are required, preferentially low-cost, to indicate the degradation stages aiming the selection of techniques to reclaim degraded areas. The purpose of this study was to analyze the variability of microbiological and chemical soil properties as indicators of desertification processes in areas with increasing levels of degradation in the semiarid region of the State of Pernambuco, Brazil. Three environments were sampled: conserved, moderately degraded and heavily degraded in Floresta, PE. Seven samples were collected from four areas per environment (28 points per environment). Soil samples were collected from the 0-10 layer in dry and rainy seasons. Soil samples were used for chemical ( $p H$, exchangeable bases, potential acidity, soil organic carbon, carbon stock, available phosphorus and cation exchange capacity), microbiological (microbial biomass carbon, basal respiration, microbial and metabolic quotients) and physical analyses (texture and bulk density). The data were analyzed using main components and $t$ test. An effect of seasonality was observed for the soil properties, especially in the degraded environment. Principal component analysis identified microbial biomass carbon, potential acidity and percent of base saturation as most sensitive to the degradation level in the rainy and dry seasons. These properties can therefore be used as indicators of soil degradation.
\end{abstract}

Index terms: Soil degradation, organic matter, soil quality.

\section{INTRODUÇÃO}

No semiárido nordestino, a degradação dos recursos naturais tem sido provocada pelo aumento da intensidade de uso do solo e redução da cobertura vegetal nativa (Menezes \& Sampaio, 2002). A retirada da caatinga, vegetação nativa nas regiões semiáridas do Nordeste, aliada a longos períodos de estiagem, provoca acentuada degradação do solo, deixando-o descoberto e exposto por mais tempo à ação dos agentes climáticos, reduzindo, consequentemente, seu potencial produtivo, causando danos muitas vezes irreversíveis ao meio (Trevisan et al., 2002; Menezes et al., 2005).

A estimativa da degradação do solo deve considerar a integração complexa de fatores químicos, físicos e biológicos, funcionalmente dinâmicos, e objetiva caracterizar o estado atual do solo, prever os caminhos de sua transformação e propor procedimentos adequados para recuperação, bem como monitorar e avaliar os impactos antropogênicos (Snakin et al., 1996; Nortcliff, 2002). Várias estratégias de avaliação da qualidade do solo têm sido propostas, destacandose as que consideram a necessidade de um conjunto numeroso de atributos do solo para a obtenção de um índice confiável de qualidade (Larson \& Pierce, 1991; Doran \& Parkin, 1994). Opondo-se a estas, existem também as que consideram que um número reduzido de atributos-chave, como a matéria orgânica do solo, pode expressar eficientemente a qualidade do solo (Gregorich et al., 1994; Seybold et al., 1998). Esta pode ser considerada um importante indicador da qualidade do solo, pois está relacionada com diversas de suas propriedades químicas, físicas e biológicas. O teor de C orgânico tem sido utilizado frequentemente como indicador-chave da qualidade do solo, tanto em sistemas agrícolas como em áreas de vegetação nativa (Silva Júnior et al., 2004; Jansen, 2005).

Os microrganismos do solo são os principais componentes do sistema de decomposição da matéria orgânica e têm papel fundamental na dinâmica de nutrientes em diferentes ecossistemas (Diaz-Raviña et al., 1993). Dentre os indicadores do solo capazes de representar a comunidade microbiana, o C microbiano destaca-se devido à sua relação com a matéria orgânica, ciclagem de nutrientes e fluxo de energia (De-Polli \& Guerra, 1999).

Atualmente, poucos são os trabalhos que envolvem variáveis químicas e microbianas de solos na região semiárida e sua utilização como indicadores de 
qualidade de áreas em processo de degradação, visando fornecer subsídios para a prevenção do processo e elaboração de estratégias de recuperação de áreas já degradadas. Assim, o objetivo deste trabalho foi estudar a variabilidade de atributos químicos e microbianos do solo, bem como a sua utilização como indicadores de processos de desertificação em áreas com níveis crescentes de degradação, na região semiárida do Estado de Pernambuco.

\section{MATERIAL E MÉTODOS}

A área utilizada neste estudo situa-se no município de Floresta-PE, localizado na Mesorregião do São Francisco Pernambucano, entre as coordenadas $8^{\circ} 36^{\prime}$ de latitude sul e de $38^{\circ} 34^{\prime}$ 'de longitude oeste. De acordo com a classificação de Köppen, o município de Floresta apresenta o clima do tipo BSw'h' e 4aTh da classificação de Gaussen, tropical quente de seca acentuada (Jacomine et al., 1973). A temperatura média anual é de $26,5^{\circ} \mathrm{C}$ e as precipitações pluviais variam em torno de $623 \mathrm{~mm}$. A evapotranspiração potencial média é de $1.646 \mathrm{~mm}$ ao ano, com déficit hídrico anual de $1.023 \mathrm{~mm}$ (EMBRAPA - Banco de dados climáticos do Brasil). As classes de solo predominantes na área de estudo são os Luvissolos, caracterizando-se por uma profundidade reduzida, presença de horizonte $\mathrm{B}$ textural de cores fortes e argila de atividade alta. Possuem alta suscetibilidade à erosão, sobretudo em áreas naturalmente degradadas (Galindo, 2007).

Os ambientes de amostragem foram definidos de acordo com áreas selecionadas por Galindo (2007), com base na interpretação preliminar de imagens de satélite e da observação, em campo, do grau de degradação dos solos (ocorrência de erosão, taxa de recobrimento, encrostamento, etc.) e da vegetação (permeabilidade, altura, ocorrência de espécies indicadoras, etc.), designando, dessa forma, três ambientes: conservado, moderadamente degradado e intensamente degradado, os quais apresentavam semelhantes características climáticas, tipos de solos, condições de relevo e altitude. Os ambientes conservados foram selecionados em áreas cercadas, raramente pastejadas, pouco erodidas, caracterizadas por vegetação de caatinga arbustiva ou arbóreoarbustiva densa; os ambientes moderadamente degradados, em áreas com vegetação de caatinga arbustiva a arbóreo-arbustiva pouco densa, apresentando erosão hídrica moderada; e os ambientes degradados, em áreas caracterizadas por vegetação de caatinga aberta, esparsa, com alta intensidade de pastejo e ocorrência de severa erosão hídrica.

Em cada ambiente selecionado foram definidas e georreferenciadas quatro áreas (Quadro 1), e em cada área o solo foi classificado de acordo com Galindo (2007), nas quais foram escolhidos sete pontos de amostragem, espaçados 10 m entre si, totalizando 84 coletas. Em cada ponto de amostragem foram coletadas amostras de solo na profundidade de $0-10 \mathrm{~cm}$, com auxílio de um anel amostrador de aço inox para melhor representação da profundidade da camada estudada, bem como amostras indeformadas para determinação da densidade do solo. Parte do solo coletado foi mantida sob refrigeração para determinação das análises microbianas.

A amostragem do solo nos ambientes estudados foi realizada em duas etapas: a primeira no período seco (outubro e novembro de 2007) e a segunda no período chuvoso (abril e maio de 2008), sendo esta última na mesma parcela do período seco, visando avaliar a variação sazonal dos ambientes em estudo.

Quadro 1. Localização geográfica e classificação do solo das quatro áreas dos ambientes conservado, moderadamente degradado e intensamente degradado, no município de Floresta-PE

\begin{tabular}{cll}
\hline Área & \multicolumn{1}{c}{ Coordenada } & \multicolumn{1}{c}{ Classificação do solo } \\
& & Ambiente Conservado \\
CF1 & UTM 24L 544805 mE e 9054570 mN & Luvissolo Crômico órtico vertissólico \\
CF2 & UTM 24L 544656 mE e 9055852 mN & Luvissolo Crômico órtico vertissólico \\
CF3 & UTM 24L 558061 mE e 9062690 mN & Luvissolo Crômico órtico vertissólico solódico \\
CF4 & UTM 24L 556975 mE e 9055112 mN & Luvissolo Crômico órtico vertissólico solódico \\
& & Ambiente moderadamente degradado \\
MF1 & UTM 24L 551370 mE e 9034294 mN & Luvissolo Crômico órtico planossólico solódico \\
MF2 & UTM 24L 556523 mE e 9055498 mN & Luvissolo Crômico órtico vertissólico sódico \\
MF3 & UTM 24L 550757 mE e 9049162 mN & Luvissolo Crômico órtico vertissólico solódico \\
MF4 & UTM 24L 554888 mE e 9049456 mN & Ambissolo Háplico Ta eutrófico léptico \\
& & Ambiente degradado \\
DF1 & UTM 24L 551386 mE e 9034294 mN & Luvissolo Crômico órtico lítico \\
DF2 & UTM 24L 550663 mE e 9049162 mN & Luvissolo Crômico órtico planossólico solódico \\
DF3 & UTM 24L 554952 mE e 9049548 mN & Luvissolo Crômico órtico vertissólico solódico vertissólico \\
DF4 & UTM 24L 552793 mE e 9050286 mN &
\end{tabular}


Foi determinada a densidade do solo pelo método do torrão parafinado, e a composição granulométrica (Quadro 2), pelo método do densímetro (Embrapa, 1997), sendo a fração areia separada por peneiramento. As análises químicas foram realizadas seguindo os métodos descritos pela Embrapa (1997): pH (1:2,5); determinação dos cátions trocáveis $\mathrm{Ca}^{2+}, \mathrm{Mg}^{2+}, \mathrm{Na}^{+} \mathrm{e}$ $\mathrm{K}^{+}$, extraídos com acetato de amônio a $\mathrm{pH}$ 7,0, dosando-

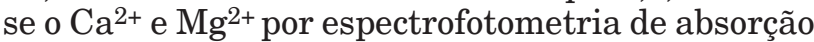
atômica e $\mathrm{Na}^{+}$e $\mathrm{K}^{+}$por fotometria de chama; $\mathrm{H}+\mathrm{Al}$ extraídos com acetato de cálcio $1 \mathrm{~mol} \mathrm{~L}^{-1}$ a pH 7,0 e determinados volumetricamente com solução de $\mathrm{NaOH} 0,025 \mathrm{~mol} \mathrm{~L}^{-1}$. O P disponível foi determinado pelo método de Bray-1 (Bray \& Kurtz, 1945) e determinado por colorimetria (Braga \& Defelipo, 1974). A determinação do C orgânico total (COT) foi feita por meio de oxidação, utilizando solução de dicromato de $\mathrm{K} 0,167 \mathrm{~mol} \mathrm{~L}^{-1}$ como agente oxidante (Yeomans \& Bremner, 1988). O estoque de $\mathrm{C}$ foi determinado utilizando-se o teor de $\mathrm{C}$ orgânico total, a densidade global do solo e a profundidade da camada de coleta. Em cada uma das camadas amostradas, ele foi estimado a partir da expressão:

$$
\text { Est } \mathrm{C}=(\mathrm{C} \times \mathrm{Ds} \times \mathrm{e}) / 10
$$

em que Est $\mathrm{C}=$ estoque de $\mathrm{C}$ em $\mathrm{Mg}$ ha-1; $\mathrm{C}$ = carbono orgânico total em $\mathrm{g} \mathrm{kg}^{-1}$; Ds = densidade do solo do horizonte estudado $\mathrm{em} \mathrm{kg} \mathrm{dm}^{-3} ; \mathrm{e}=$ profundidade de coleta $(\mathrm{cm})$.

Quadro 2. Granulometria e densidade dos solos nos ambientes conservado, moderadamente degradado e intensamente degradado, na profundidade de $0-10 \mathrm{~cm}$

\begin{tabular}{llllc}
\hline \multicolumn{1}{c}{ Ambiente } & Areia & Silte & Argila & Ds \\
\hline & & $\mathrm{g} \mathrm{kg}^{-1}$ & & $\mathrm{~kg} \mathrm{dm}^{-3}$ \\
\cline { 2 - 4 } & & & & \\
Conservado & 531 & 132 & 337 & 1,65 \\
Moderadamente degradado & 546 & 136 & 318 & 1,69 \\
Intensamente degradado & 537 & 109 & 353 & 1,70 \\
\hline
\end{tabular}

Realizaram-se as seguintes análises microbianas: C da biomassa microbiana do solo (C-BMS) e respiração basal do solo. O C-BMS foi determinado pelo método da irradiação-extração (Vance et al., 1987), utilizando o forno micro-ondas de potência igual a $900 \mathrm{~W}$ e frequência de $2.450 \mathrm{MHz}$, conforme método descrito por Islam \& Weil (1998). Foi utilizado como agente oxidante o permanganato de potássio e, na determinação do C contido nos extratos, empregou-se um método colorimétrico (Bartlett \& Ross, 1988). Para determinação da respiração basal do solo, foram tomadas amostras, em triplicata, umedecidas a $80 \%$ da capacidade de retenção de água do solo e armazenadas em potes de vidro, juntamente com uma amostra de $25 \mathrm{~mL}$ da solução de $\mathrm{NaOH} 0,1 \mathrm{~mol} \mathrm{~L}^{-1}$. Foi estimado o $\mathrm{CO}_{2}$ liberado pela respiração, utilizando $\mathrm{NaOH} 0,1 \mathrm{~mol} \mathrm{~L}^{-1}$ e titulando com HCl $1 \mathrm{~mol} \mathrm{~L}^{-1} \mathrm{e}$ fenolftaleína como indicador, após $72 \mathrm{~h}$ de incubação a $25-28^{\circ} \mathrm{C}$. Calculou-se o quociente metabólico $\left(q \mathrm{CO}_{2}\right)$ obtido pela razão entre a respiração basal do solo por unidade de C-BMS (Anderson \& Domsch, 1985) e o quociente microbiano ( $q \mathrm{MIC}$ ) obtido pela razão entre o C-BMS e o COT do solo.

Foi aplicado o teste $\mathrm{t}$ para avaliar o efeito da sazonalidade (períodos seco e chuvoso) nas variáveis analisadas, sendo o SAEG 9.1 (UFV, 2007) o software utilizado. Foram empregadas técnicas de análise multivariada como ferramenta adicional, especificamente a Análise de Componentes Principais (STATISTICA, 2004), para distinção dos ambientes em função do grau de degradação.

\section{RESULTADOS E DISCUSSÃO}

O efeito da sazonalidade foi percebido apenas em alguns atributos do solo (Quadro 3). Nos três ambientes (conservado, moderadamente degradado e intensamente degradado) há, em alguns atributos, diferença significativa entre as médias dos períodos seco e chuvoso. Essa inferência traz o comportamento dos ambientes em função da condição climática da região, uma vez que os ambientes semiáridos apresentam como principal limitação a deficiência hídrica.

No ambiente conservado, houve alterações tanto de ordem química quanto microbiana. Para os atributos químicos, destaque merece ser dado à redução da CTC potencial do solo do período seco para o chuvoso, tendo como principal influenciador desse comportamento o teor de $\mathrm{Mg}$, que apresentou redução de aproximadamente $50 \%$ (Quadro 3). Isso pode ser atribuído à maior demanda desse nutriente pelas plantas, uma vez que a presença de água pode ter facilitado sua disponibilidade, e, com essa condição climática, o ambiente tornou-se favorável ao crescimento das plantas e consequente maior demanda desse macronutriente. Além disso, pode-se afirmar que a disponibilidade de nutrientes é normalmente alterada pelas mudanças edafoclimáticas do ambiente (Pavinato et al., 2009). Já ao se observarem as médias do teor de C-BMS e os quocientes $q \mathrm{MIC}$ e $q \mathrm{CO}_{2}$, nos períodos seco e chuvoso do mesmo ambiente conservado, percebe-se redução significativa para C-BMS e, consequentemente, do $q \mathrm{MIC}(2,56 \%$ no período seco e $1,60 \%$ no chuvoso) e aumento do $q \mathrm{CO}_{2}$. Possivelmente essa redução tenha ocorrido em razão da mineralização da biomassa microbiana, reduzindo assim sua comunidade. Alvarenga et al. (1999) obtiveram valores de $q \mathrm{MIC}$ que variaram de $3,08 \%$ no Cerrado até $1,34 \%$ em pasto nativo, estando de acordo com a percentagem proposta por Jenkinson \& Ladd (1981), que consideram normal que 1 a $4 \%$ do $\mathrm{C}$ total do solo corresponda ao componente microbiano. O aumento do $q \mathrm{CO}_{2}$ é 
Quadro 3. Atributos químicos e microbianos dos períodos seco e chuvoso, nos ambientes conservado, moderadamente degradado e intensamente degradado

\begin{tabular}{|c|c|c|c|c|c|c|}
\hline \multirow{3}{*}{ Atributo } & \multicolumn{6}{|c|}{ Ambiente } \\
\hline & \multicolumn{2}{|c|}{ Conservado } & \multicolumn{2}{|c|}{ Moderadamente degradado } & \multicolumn{2}{|c|}{ Degradado } \\
\hline & Seco & Chuvoso & Seco & Chuvoso & Seco & Chuvoso \\
\hline COT (dag kg $\left.{ }^{-1}\right)$ & 1,377 & $1,397^{\mathrm{ns}}$ & 1,092 & $0,944^{\mathrm{ns}}$ & 0,581 & $0,845^{*}$ \\
\hline Est $\mathrm{C}\left(\mathrm{Mg} \mathrm{ha}^{-1}\right)$ & 21,682 & $23,164^{\mathrm{ns}}$ & 17,974 & $15,644^{\mathrm{ns}}$ & 9,699 & $14,009^{*}$ \\
\hline $\mathrm{pH}$ & 7,116 & $7,116^{\text {ns }}$ & 7,464 & $7,339^{\text {ns }}$ & 7,419 & $7,404^{\mathrm{ns}}$ \\
\hline $\mathrm{P}\left(\mathrm{mg} \mathrm{kg}^{-1}\right)$ & 5,550 & $4,322^{\text {ns }}$ & 6,178 & $4,088^{*}$ & 6,087 & $4,207^{\mathrm{ns}}$ \\
\hline $\mathrm{Ca}^{2+}\left(\mathrm{cmol}_{\mathrm{c}} \mathrm{dm}^{-3}\right)$ & 11,215 & $11,058^{\mathrm{ns}}$ & 11,282 & $10,256^{\mathrm{ns}}$ & 11,170 & $13,319^{*}$ \\
\hline $\mathrm{Mg}^{2+}\left(\mathrm{cmol}_{\mathrm{c}} \mathrm{dm}^{-3}\right)$ & 8,575 & $4,575^{*}$ & 5,500 & $5,393^{\text {ns }}$ & 6,277 & $5,803^{\text {ns }}$ \\
\hline $\mathrm{Na}^{+}\left(\mathrm{cmol}_{\mathrm{c}} \mathrm{dm}^{-3}\right)$ & 0,098 & $0,076^{\mathrm{ns}}$ & 0,119 & $0,172^{\text {ns }}$ & 0,325 & $0,316^{\text {ns }}$ \\
\hline $\mathrm{K}^{+}\left(\mathrm{cmol}_{\mathrm{c}} \mathrm{dm}^{-3}\right)$ & 0,607 & $0,477^{*}$ & 0,358 & $0,297^{\mathrm{ns}}$ & 0,252 & $0,217^{*}$ \\
\hline $\mathrm{H}+\mathrm{Al}\left(\mathrm{cmol}_{\mathrm{c}} \mathrm{dm}^{-3}\right)$ & 1,783 & $2,216^{*}$ & 0,257 & $0,643^{*}$ & 0,242 & $0,391^{*}$ \\
\hline $\mathrm{CTC}_{\mathrm{pot}}\left(\mathrm{cmol}_{\mathrm{c}} \mathrm{dm}^{-3}\right)$ & 22,278 & $18,402^{*}$ & 17,517 & $16,762^{\text {ns }}$ & 18,266 & $20,046^{\mathrm{ns}}$ \\
\hline Resp. Basal $\left(\mathrm{CO}_{2}(\mathrm{mg}) / \mathrm{kgSS} / \mathrm{hora}\right)$ & 3,253 & $3,311^{\mathrm{ns}}$ & 1,988 & $1,857^{\mathrm{ns}}$ & 2,125 & $1,311^{*}$ \\
\hline C-BMS $\left(\mathrm{mg} \mathrm{kg}^{-1}\right)$ & 332,832 & $199,256^{*}$ & 109,655 & 135,865 * & 64,324 & $67,170^{\mathrm{ns}}$ \\
\hline$q \mathrm{MIC}(\%)$ & 2,575 & $1,596^{*}$ & 1,024 & $1,591^{*}$ & 1,170 & $0,794^{*}$ \\
\hline$q \mathrm{CO}_{2}\left(\mathrm{mg} \mathrm{CO}_{2} \mathrm{mg}^{-1} \mathrm{Cmic} \mathrm{dia}^{-1}\right)$ & 0,013 & $0,030^{*}$ & 0,021 & $0,021^{\mathrm{ns}}$ & 0,042 & $0,026^{*}$ \\
\hline
\end{tabular}

*: diferença significativa a $5 \%$; ns: diferença não significativa (teste t de Student). COT: carbono orgânico total; EstC - Estoque de carbono; C-BMS: carbono da biomassa microbiana do solo; $q \mathrm{MIC}$ : quociente microbiano; $q \mathrm{CO}_{2}$ : quociente metabólico.

justamente uma resposta à mineralização da biomassa microbiana, uma vez que relaciona o quanto de $\mathrm{CO}_{2}$ foi liberado devido à mineralização.

Para o ambiente moderadamente degradado, menor quantidade de atributos químicos e microbianos variou sua média em função da sazonalidade do ambiente. Ênfase pode ser dada ao aumento da atividade microbiana no período chuvoso, representada pelo C-BMS e $q \mathrm{MIC}$. Como esse ambiente apresentase em processo de degradação, pode-se inferir que uma sucinta melhoria da condição hídrica foi capaz de proporcionar melhor condição ao desenvolvimento de microrganismos do solo, condição essa que não havia no período seco.

A maior quantidade de atributos do solo que variaram em função da sazonalidade foi detectada no ambiente degradado (Quadro 3). Dentre os atributos químicos que apresentaram maior média no período chuvoso, destaque merece ser dado ao teor de $\mathrm{Ca}$, que apresentou aumento de aproximadamente $50 \%$. Pavinato et al. (2009) observaram que há incremento na disponibilidade de $\mathrm{Ca}$, geralmente, em consequência da disponibilização desse nutriente dos resíduos em decomposição; logo, esse comportamento também pode ser consequência de o ambiente apresentar, em função de distúrbios nutricionais causados pela degradação, rápida resposta a mudanças hídricas no solo, além de a severa erosão hídrica remover as camadas superficiais do solo, expondo a camada subsuperficial, mais rica em Ca. Esse fato foi observado por Galindo et al. (2008), que constataram a remoção total do horizonte A dos perfis de algumas das áreas semiáridas mais severamente degradadas de Jataúba-PE.
Igual destaque pode ser dado ao COT e, consequentemente, ao EstC, visto que esses atributos aumentaram cerca de $45 \%$. Esse aporte de $\mathrm{C}$ ao solo deveu-se, provavelmente, ao crescimento de herbáceas, que encerram seu ciclo rapidamente, contribuindo para significativo aumento do teor de C, principalmente devido à decomposição de raízes finas. Salcedo et al. (1999), citados por Salcedo \& Sampaio (2008), observaram que estoques de raízes de até $5 \mathrm{~mm}$ de espessura e até $30 \mathrm{~cm}$ de profundidade variaram de 3 a 8 t ha $^{-1}$ em áreas de caatinga do Estado da Paraíba. Pillon et al. (2007), em experimento consorciado sob plantio direto no RS, observaram que o maior potencial no incremento de $\mathrm{C}$ foi proporcionado pela consorciação gramínea/leguminosa no inverno. O predomínio de gramíneas na estação chuvosa, como o capim-panasco (Aristida sp.) nesses sítios degradados, foi observado por Galindo (2007). Essa melhor condição, em razão principalmente da presença de água, reduziu significativamente o $q \mathrm{CO}_{2}$ no período chuvoso (Quadro 3). As condições ambientais para melhor desenvolvimento da microbiota tornam-se evidentes no período chuvoso; logo, o ambiente degradado tende a apresentar comportamento mais próximo ao do conservado, uma vez que os organismos, como mecanismo de defesa, liberam quantidade mínima de $\mathrm{CO}_{2}$, reservando assim energia (Wardle, 1994). Em geral, baixo $q \mathrm{CO}_{2}$ indica economia na utilização de energia e, supostamente, reflete um ambiente mais estável ou mais próximo do seu estado de equilíbrio; ao contrário, valores elevados são indicativos de ecossistemas submetidos a alguma condição de estresse ou de distúrbio (Anderson \& Domsch, 1985). 
Como ferramenta para a distinção dos ambientes estudados, foram geradas duas componentes principais (Fator 1 e Fator 2) para os atributos químicos (COT, pH, P, $\mathrm{Ca}^{2+}, \mathrm{Mg}^{2+}, \mathrm{Na}^{+}, \mathrm{K}^{+}, \mathrm{H}+\mathrm{Al}$ e valor $\mathrm{V}$ ) e microbianos (RB, C-BMS, $q \mathrm{MIC}$ e $q \mathrm{CO}_{2}$ ) em conjunto, tanto para o período seco como para o chuvoso. A partir da relação entre essas componentes, foram formados diagramas de ordenação bidimensionais, para visualização da distinção dos três ambientes, e diagramas de projeção de vetores, para os atributos do solo que mais influenciaram nessa distinção, mostrando, assim, maior sensibilidade (Figura 1a). A visualização dos diagramas permite perceber que os ambientes estudados formaram três grupos, que, mesmo com pontos de sobreposição, tendem a se distinguir no diagrama de ordenação (Figura 1b).

Essa análise permite confirmar a influência dos atributos químicos e microbianos, nessa camada superficial, em diferenciar os ambientes quanto ao nível de degradação. O Fator 1, gerado para os atributos do período seco (Figura 1a,b), explicou $35,48 \%$ da variação total dos atributos estudados, e os maiores coeficientes de correlação $(\geq|0,70|)$ foram identificados para as variáveis C-BMS, $q \mathrm{MIC}$, COT, $\mathrm{H}+\mathrm{Al}$ e valor V (Quadro 4), ou seja, esses atributos foram mais sensíveis na distinção dos ambientes. Isso pode ser visualizado no diagrama de projeção dos vetores, onde esses atributos apresentam-se mais distantes do eixo do Fator 1 (Figura 1a). Já para o
Fator 2, onde a variância explicada foi menor (17,47 \%), apenas o Ca foi identificado como atributo sensível na distinção dos ambientes, apresentando maior distância de seu vetor em relação ao eixo do Fator 2 (Figura 1a). Ao analisar os mesmos atributos no período chuvoso, pode-se observar que as duas componentes principais (Fatores 1 e 2) explicaram 45,93 \% da variação total dos atributos (Quadro 4), sendo os maiores coeficientes de correlação apresentados apenas para o C-BMS, $\mathrm{H}+\mathrm{Al}$ e valor $\mathrm{V}$ (Figura $1 \mathrm{c}$ ) .

A interpretação dos coeficientes de correlação (Quadro 4) segue o mesmo raciocínio de uma análise de correlação linear simples, podendo-se inferir que os atributos que apresentam coeficiente de correlação negativo (C-BMS, qMIC, COT, $\mathrm{H}+\mathrm{Al}$ e $\mathrm{Ca}^{2+}$ ) decrescem com o aumento do nível de degradação dos ambientes. Wick et al. (1998), utilizando a análise de componentes principais, observaram que as variáveis relacionadas à dinâmica de nutrientes da matéria orgânica do solo contribuíram para explicar mais de $80 \%$ da variância total dos dados, confirmando que variáveis como C-BMS e COT podem ser utilizadas como indicadores sensíveis na avaliação da qualidade do solo. O único atributo que diretamente correlaciona-se com a degradação do solo é a saturação por bases (V); esse comportamento pode ser explicado pela concentração dos sais solúveis no solo (salinização/ sodicidade), que pode ser característico de ambientes em processo de degradação.
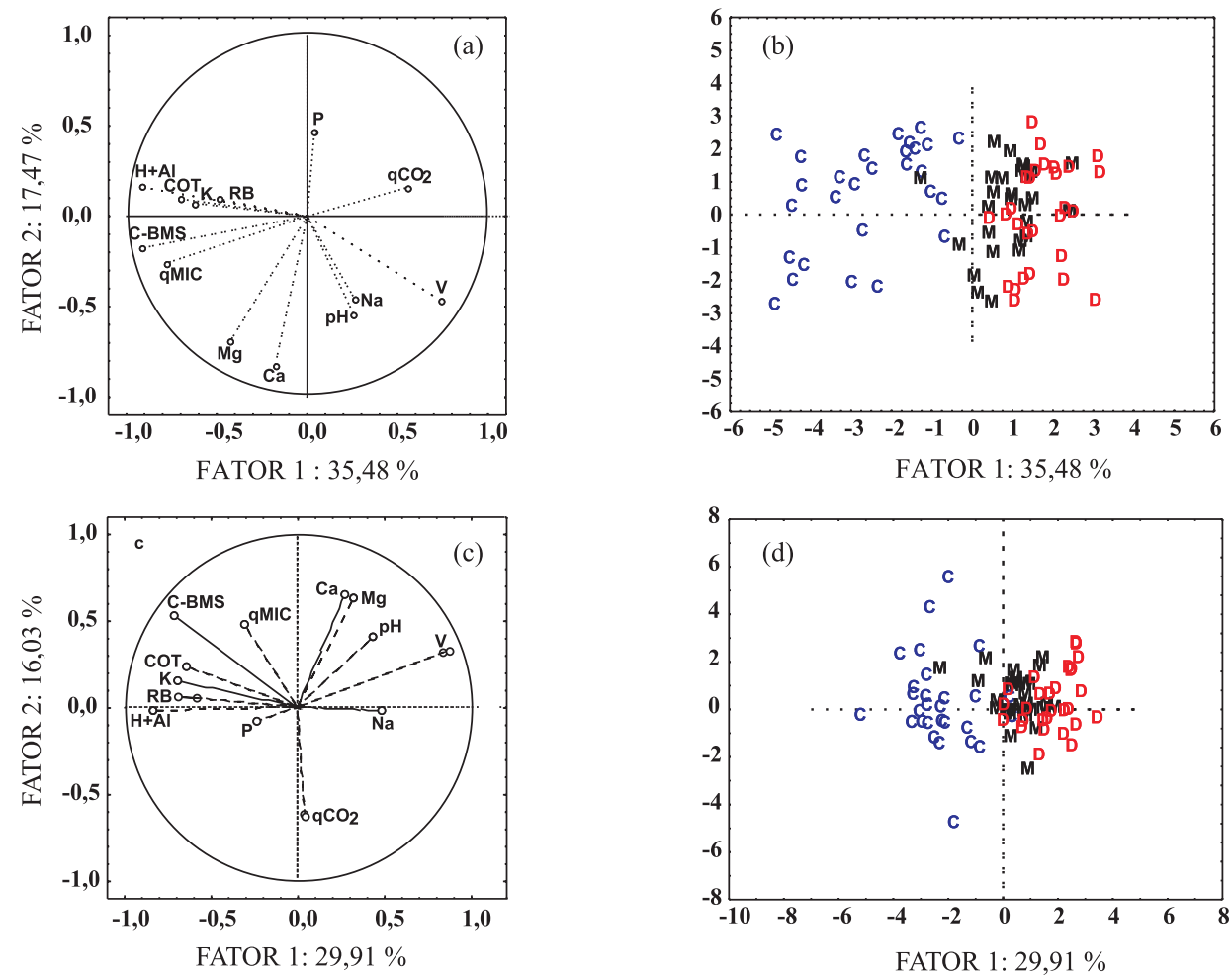

Figura 1. Diagrama de projeção dos vetores dos atributos químicos e microbianos durante os períodos seco (a) e chuvoso (c) e diagrama de ordenação dos componentes principais para os ambientes conservado (C), moderadamente degradado (M) e degradado (D), durante os períodos seco (b) e chuvoso (d). 
Quadro 4. Coeficientes de correlação dos componentes principais (Fatores 1 e 2) para os atributos químicos e microbianos, em função do nível de degradação dos ambientes, durante os períodos seco e chuvoso

\begin{tabular}{|c|c|c|c|c|}
\hline \multirow{3}{*}{ Variável } & \multicolumn{4}{|c|}{ Período } \\
\hline & \multicolumn{2}{|c|}{ Seco } & \multicolumn{2}{|c|}{ Chuvoso } \\
\hline & Fator $1^{(1)}$ & Fator $2^{(1)}$ & Fator $1^{(1)}$ & Fator $2^{(1)}$ \\
\hline $\mathrm{RB}$ & $-0,490098$ & 0,094028 & $-0,585375$ & 0,057132 \\
\hline C-BMS & $-0,918413$ & $-0,177052$ & $-0,713185$ & 0,536795 \\
\hline qMIC & $-0,775636$ & $-0,258186$ & $-0,307145$ & 0,484163 \\
\hline $\mathrm{qCO}_{2}$ & 0,551888 & 0,158510 & 0,039595 & $-0,610911$ \\
\hline COT & $-0,702056$ & 0,096162 & $-0,638889$ & 0,235820 \\
\hline $\mathrm{pH}$ & 0,257407 & $-0,543274$ & 0,431468 & 0,406865 \\
\hline $\mathrm{P}$ & 0,039731 & 0,469051 & $-0,233396$ & $-0,073926$ \\
\hline $\mathrm{Ca}^{2+}$ & $-0,177814$ & $-0,825444$ & 0,270523 & 0,654824 \\
\hline $\mathrm{Mg}^{2+}$ & $-0,426025$ & $-0,691964$ & 0,324389 & 0,632004 \\
\hline $\mathrm{Na}^{+}$ & 0,260500 & $-0,452926$ & 0,477926 & $-0,015198$ \\
\hline $\mathrm{K}^{+}$ & $-0,627059$ & 0,073033 & $-0,693471$ & 0,159461 \\
\hline $\mathrm{H}+\mathrm{Al}$ & $-0,917243$ & 0,161257 & $-0,837338$ & $-0,015456$ \\
\hline $\mathrm{V}$ & 0,739415 & $-0,467151$ & 0,838381 & 0,321785 \\
\hline Variância Total (\%) & 35,48 & 17,47 & 29,91 & 16,03 \\
\hline Variância acumulada (\%) & 35,48 & 52,95 & 29,91 & 45,93 \\
\hline
\end{tabular}

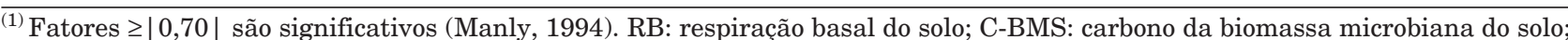
qMIC: quociente microbiano; $q \mathrm{CO}_{2}$ : quociente metabólico; COT: carbono orgânico total.

\section{CONCLUSÕES}

1. Verificou-se o efeito da sazonalidade para os atributos do solo, sendo essa mudança mais percebida no ambiente degradado.

2. As análises de componentes principais demonstraram que alguns atributos químicos e microbianos são mais sensíveis ao avanço da degradação, como o $\mathrm{C}$ da biomassa microbiana do solo, a acidez potencial e o valor $\mathrm{V}$, tanto no período seco como no chuvoso.

3. Os atributos mais sensíveis (C-BMS, qMIC, $\mathrm{COT}, \mathrm{Ca}, \mathrm{H}+\mathrm{Al}$ e valor $\mathrm{V}$ ) podem ser utilizados como indicadores do nível de degradação do solo.

\section{AGRADECIMENTOS}

À Universidade Federal Rural de Pernambuco UFRPE, à CAPES e ao CNPq, pelo apoio técnico e financeiro.

\section{LITERATURA CITADA}

ANDERSON, J.P.E. \& DOMSCH, K.H. Determination of ecophysiological maintenance carbon requirements of soil microorganisms in dormant state. Biol. Fert. Soils, 1:81$89,1985$.
ALVARENGA, M.I.N.; SIQUEIRA, J.O. \& DAVIDE, A.C. Teor de carbono, biomassa microbiana, agregação e micorriza em solos de cerrado com diferentes usos. Ci. Agrotec., 23:617-625, 1999.

BARTLETT, R.J. \& ROSS, S.D. Colorimetric determination of oxidizable carbon in acid soil solutions. Soil Sci. Soc. Am. J., 52:1191-1192, 1988.

BRAGA, J.M. \& DEFELIPO, B.V. Determinação espectrofotométrica de fósforo em extratos de solo e material vegetal. R. Ceres, 21:73-85, 1974.

BRAY, R.H. \& KURTZ, L.T. Determination of total, organic and available forms of phosphorus in soils. Soil Sci., 59:3945, 1945.

DE-POLLI, H. \& GUERRA, J.G.M. C, N e P na biomassa microbiana do solo. In: SANTOS, G.A. \& CAMARGO, F.A.O., eds. Fundamentos da matéria orgânica do solo: Ecossistemas tropicais e subtropicais. Porto Alegre, Gênesis, 1999. p.389-412.

DÍAZ-RAVIÑA, M.; ACEA, M.J. \& CARBALLAS, T. Microbial biomass and its contribution to nutrient concentrations in forest soils. Soil Biol. Biochem., 25:25-31, 1993.

DORAN, J.W. \& PARKIN, T.B. Defining and assessing soil quality. In: DORAN, J.W.; COLEMAN, D.C.; BEZDICEK, D.F. \& STEWART, B.A., eds. Defining soil quality for a sustainable environment. Madison, Soil Science Society America, 1994. v.35. p.3-22.

EMPRESA BRASILEIRA DE PESQUISA AGROPECUÁRIA EMBRAPA. Centro Nacional de Pesquisa de Solos. Manual de métodos de análise de solos. Rio de Janeiro, 1997. 212p. 
EMPRESA BRASILEIRA DE PESQUISA AGROPECUÁRIA EMBRAPA. Banco de dados climáticos do Brasil Disponível em: (http://www.bdclima.cnpm.embrapa.br/ resultados/balanco.php?UF $=\& \mathrm{COD}=141$ )

GALINDO, I.C.L. Relações solo-vegetação em áreas sob processo de desertificação no Estado de Pernambuco. Recife, Universidade Federal de Pernambuco, 2007. 255p. (Tese de Doutorado)

GALINDO, I.C.L.; RIBEIRO, M.R.; SANTOS, M.F.A.V.; LIMA, J.F.W.F. \& FERREIRA, R.F.A.L. Relações solo-vegetação em áreas sob processo de desertificação no município de Jataúba, PE. R. Bras. Ci. Solo, 32:1283-1296, 2008

GREGORICH, E.G.; CARTER, M.R.; ANGERS, D.A.; MONREAL, C.M. \& ELLERT, B.H. Towards a minimum data set to assess soil organic matter quality in agricultural soils. Canadian J. Soil Sci., 74:367-375, 1994.

ISLAM, K.R. \& WEIL, R.R. Microwave irradiation of soil for routine measurement of microbial biomass carbon. Biol. Fert. Soils, 27:408-416, 1998.

JACOMINE, P.K.T.; CAVALCANTI, A.C.; BURGOS, N. \& PESSOA, S.C.P. Levantamento exploratório reconhecimento de solos do Estado de Pernambuco. Recife, MA/DNPEA - SUDENE/DRN, 1973. v.1. (Boletim Técnico, 26)

JANSEN, H.H. Soil carbon: A measure of ecosystem response in a changing world? Canadian J. Sci., 85:467-480, 2005.

JENKINSON, D.S. \& LADD, J.N. Microbial biomass in soil: Measurements and turnover. In: PAUL, E.A. \& LADD, J. N., eds. Soil biochem. 5.ed. New York, Marcel Dekker, 1981. p.415-471.

LARSON, W.E. \& PIERCE, F.J. Conservation and enhancement of soil quality. In: EVALUATION ON FOR SUSTAINABLE LAND MANAGEMENT IN THE DEVELOPING WORLD, Bangkok, 1992. IBSRAM Proceedings... Bangkok, Int. Board for Soil Res. And Management,1991 p. $175-203$.

MANLY, B.F.J. Multivariate statistical methods. 2.ed. London, Chapman \& Hall, 1994. 215p.

MENEZES, R.S.C. \& SAMPAIO, E.V.S.B. Simulação dos fluxos e balanços de fósforo em uma unidade de produção agrícola familiar no semi-árido paraibano. In: SILVEIRA, L.M.; PETERSEN, P. \& SABOURIN, E., orgs. Agricultura familiar e agroecologia no semi-árido: Avanços a partir do Agreste da Paraíba. Rio de Janeiro, AS-PTA, 2002. p.249-260.

MENEZES, R.C.S.; GARRIDO, M.S. \& PEREZ M., A.M. Fertilidade dos solos no semi-árido. In: CONGRESSO BRASILEIRO DE CIÊNCIA DO SOLO, 30., Recife, 2005. Palestras... Recife, UFRPE/SBCS, 2005. CD-ROM.

NORTCLIFF, S. Standardisation of soil quality attributes. Agric. Ecosyst. Environ., 88:161-168, 2002.

PAVINATO, P.S.; MERLIN, A. \& ROSOLEM, C.A. Disponibilidade de cátions no solo alterada pelo sistema de manejo. R. Bras. Ci. Solo, 33:1031-1040, 2009.
PILLON, C.N.; SCIVITTARO, W.B.; POTES, M.L.; MORAES, C.S.; MICHELS, G.H. \& PEREIRA, J.S. Acúmulo de carbono orgânico por sistemas de cultura sob plantio direto em terras baixas. R. Bras. Agroecol., 2:1040-1043, 2007.

SALCEDO, I.H. \& SAMPAIO, E.V.S.B. Matéria orgânica do solo no bioma caatinga. In: SANTOS, G.A.; SILVA, L.S.; CANELLAS, L.P. \& CAMARGO, F.A.O., eds. Fundamentos da matéria orgânica do solo: Ecossistemas tropicais e subtropicais. 2.ed. Porto Alegre, Metrópole, 2008. p.419-441.

SALCEDO, I.H.; LEITE, L.; VASCONCELOS, E.; SOUZA, F. \& SAMPAIO, E.V.S.B. Produção de raízes finas sob vegetação de caatinga. In: WORKSHOP SOBRE SISTEMA RADICULAR: METODOLOGIAS E ESTUDOS DE CASO, Aracajú,1999. Anais... Aracajú, Embrapa, 1999. p.139-152.

SEYBOLD, C.A.; HERRICK, J.E. \& BREDJA, J.J. Soil resilience: A fundamental component of soil quality. Soil Sci., 164: 224-233, 1998.

SILVA JÚNIOR, A.M.; BORGES, E.N.; SOUZA, M.A.; SILVA SOUSA, G.M.;GUIMARÃES, E.C. \& BORGES, E.V.S. Carbono orgânico em diferentes sistemas de manejo no Triângulo Mineiro. In.: REUNIÃO BRASILEIRA DE MANEJO E CONSERVAĈ̃O DO SOLO E DA ÁGUA, 15., Santa Maria, 2004. Anais... Santa Maria, Universidade de Santa Maria, 2004. CD-ROM.

SNAKIN, V.V.; KRECHETOV, P.P.; KUZOVNIKOVA, T.A.; ALYABINA, I.O.; GUROV, A.F. \& STEPICHEV, A.V. The system of assessment of soil degradation. Soil Technol., 8:331-343, 1996 .

STATISTICA (data analysis software system), versão 7.0, StatSoft (www.statsoft.com), 2004.

TREVISAN, R.; MATTOS, M.L.T. \& HERTER, F.G. Atividade microbiana em Argissolo Vermelho-Amarelo distrófico coberto com aveia preta (Avena sp.) no outono, em um pomar de pessegeiro. Ci. Rural, 7:2:83-89, 2002.

UNIVERSIDADE FEDERAL DE VIÇOSA - UFV. SAEG 9.1: Sistema de análises estatística. Viçosa, MG, Fundação Arthur Bernardes, 2007. CD-ROM.

VANCE, E.D.; BROOKES, P.C. \& JENKINSON, D.S. An extraction method for measuring soil microbial biomass. Soil Biol. Biochem., 19:703-707, 1987.

WARDLE, D.A. Metodologia para quantificação da biomassa microbiana do solo. In: HUNGRIA, M. \& ARAÚJO, R.S., eds. Manual de métodos empregados em estudos de microbiologia agrícola. Brasília, Embrapa-SPI, 1994. 542p. (Embrapa/CNPAF. Documentos, 46)

WICK, B.; KÜHNE, R.F. \& VLEK, P.L.G. Soil microbiological parameters as indicators of soil quality under improved fallow management systems in south-western Nigeria. Plant Soil, 202:97-107, 1998.

YEOMANS, J.C. \& BREMNER, J.M. A rapid and precise method for routine determination of organic carbon in soil. Comm. Soil Sci. Plant Anal., 19:1467-1476, 1988. 